Wei-Guo Duan, Yi-Peng Guo, Long Lin* and Wei-Na Wu

\title{
Crystal structure of dichlorido-( $\mathrm{N}^{\prime}$-(1-(3-ethylpyrazin-2-yl)ethylidene)-4- methoxybenzohydrazide- $\left.\mathrm{K}^{3} \mathrm{~N}, \mathrm{~N}^{\prime}, \mathrm{O}\right)$ cadmium(II), $\mathrm{C}_{16} \mathrm{H}_{18} \mathrm{~N}_{4} \mathrm{O}_{2} \mathrm{Cl}_{2} \mathrm{Cd}$
}

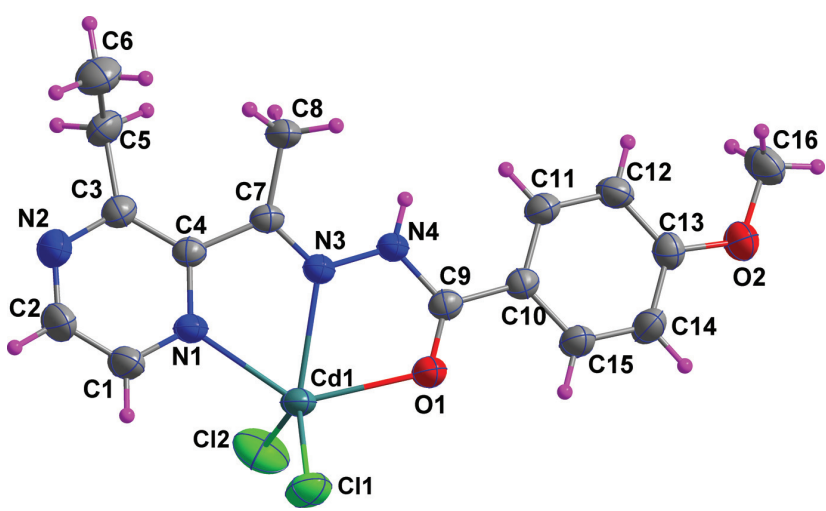

https://doi.org/10.1515/ncrs-2019-0608

Received August 21, 2019; accepted October 2, 2019; available online October 18, 2019

\section{Abstract \\ $\mathrm{C}_{16} \mathrm{H}_{18} \mathrm{~N}_{4} \mathrm{O}_{2} \mathrm{Cl}_{2} \mathrm{Cd}$, monoclinic, $P 2_{1} / n$ (no. 14), $a=9.833$ (2) $\AA$, $b=17.404(4) \AA, \quad c=11.694(3) \AA, \quad \beta=112.901(3)^{\circ}$, $V=1843.5(8) \AA^{3}, Z=4, R_{\mathrm{gt}}(F)=0.0280, w R_{\text {ref }}\left(F^{2}\right)=0.0728$, $T=296(2) \mathrm{K}$.}

\section{CCDC no.: 1957183}

The crystal structure is shown in the figure. Table 1 contains crystallographic data and Table 2 contains the list of the atoms including atomic coordinates and displacement parameters.

\section{Source of material}

4-Methoxybenzohydrazide ( $0.166 \mathrm{~g}, 1 \mathrm{mmol})$ and 2-acetopyrazine $(0.122 \mathrm{~g}, 1 \mathrm{mmol})$ were dissolved in methanol $(20 \mathrm{~mL})$. The reaction mixture was refluxed for $1 \mathrm{~h}$ and cooled to room temperature. Then cadmium(II) chloride $(0.183 \mathrm{~g}$, $1 \mathrm{mmol}$ ) was added. After stirring for $1 \mathrm{~h}$, the mixture was

*Corresponding author: Long Lin, School of Materials Science and Engineering, Henan Polytechnic University, Jiaozuo 454000, P.R. China, e-mail: linlong@hpu.edu.cn. https://orcid.org/00000003-1645-5600

Wei-Guo Duan: School of Mathematics and Physics, Weinan Normal University, Weinan 714099, P.R. China

Yi-Peng Guo: School of Materials Science and Engineering, Henan Polytechnic University, Jiaozuo 454000, P. R. China

Wei-Na Wu: College of Chemistry and Chemical Engineering, Henan Polytechnic University, Jiaozuo 454000, P.R. China. https://orcid.org/0000-0002-0874-0703
Table 1: Data collection and handling.

\begin{tabular}{ll}
\hline Crystal: & Yellow block \\
Size: & $0.15 \times 0.12 \times 0.10 \mathrm{~mm}$ \\
Wavelength: & Mo $K \alpha$ radiation $(0.71073 \AA$ A $)$ \\
$\mu:$ & $1.49 \mathrm{~mm}^{-1}$ \\
Diffractometer, scan mode: & Bruker APEX-II, $\varphi$ and $\omega$-scans \\
$\theta_{\max }$, completeness: & $25^{\circ},>99 \%$ \\
$N(h k l)_{\text {measured }}, N\left(h k l l_{\text {unique }}, R_{\text {int }}:\right.$ & $15734,3244,0.028$ \\
Criterion for $I_{\text {obs }}, N(h k l)_{\text {gt }}:$ & $I_{\text {obs }}>2 \sigma\left(I_{\text {obs }}\right), 2958$ \\
$N(\text { param })_{\text {refined }}:$ & 229 \\
Programs: & Bruker programs [1], SHELX [2]
\end{tabular}

Table 2: Fractional atomic coordinates and isotropic or equivalent isotropic displacement parameters $\left(\AA^{2}\right)$.

\begin{tabular}{lrrrr}
\hline Atom & $\boldsymbol{x}$ & $\boldsymbol{y}$ & $\boldsymbol{z}$ & $\boldsymbol{U}_{\text {iso }}{ }^{*} \boldsymbol{U}_{\text {eq }}$ \\
\hline Cd1 & $0.47352(2)$ & $0.55740(2)$ & $0.23571(2)$ & $0.03739(10)$ \\
Cl1 & $0.25031(9)$ & $0.48710(5)$ & $0.19936(9)$ & $0.0560(2)$ \\
Cl2 & $0.53495(14)$ & $0.66448(6)$ & $0.37506(10)$ & $0.0720(3)$ \\
O1 & $0.4117(2)$ & $0.61886(13)$ & $0.0430(2)$ & $0.0481(5)$ \\
O2 & $0.4183(3)$ & $0.82675(14)$ & $-0.3953(2)$ & $0.0546(6)$ \\
N1 & $0.6492(3)$ & $0.46230(14)$ & $0.3418(2)$ & $0.0361(5)$ \\
N2 & $0.8846(3)$ & $0.38111(18)$ & $0.5031(3)$ & $0.0570(8)$ \\
N3 & $0.6558(3)$ & $0.54161(13)$ & $0.1564(2)$ & $0.0324(5)$ \\
N4 & $0.6338(3)$ & $0.58158(14)$ & $0.0496(2)$ & $0.0352(5)$ \\
H4 & 0.6967 & 0.5798 & 0.0152 & $0.042^{*}$ \\
C1 & $0.6408(4)$ & $0.42534(19)$ & $0.4377(3)$ & $0.0476(8)$ \\
H1 & 0.5539 & 0.4275 & 0.4516 & $0.057^{*}$ \\
C2 & $0.7582(4)$ & $0.3840(2)$ & $0.5167(3)$ & $0.0599(10)$ \\
H2 & 0.7482 & 0.3572 & 0.5818 & $0.072^{*}$ \\
C3 & $0.8971(3)$ & $0.41819(17)$ & $0.4059(3)$ & $0.0392(7)$ \\
C4 & $0.7745(3)$ & $0.45736(15)$ & $0.3210(3)$ & $0.0317(6)$ \\
C5 & $1.0497(3)$ & $0.4128(2)$ & $0.4054(3)$ & $0.0466(8)$ \\
H5A & 1.1222 & 0.4201 & 0.4892 & $0.056^{*}$ \\
H5B & 1.0628 & 0.4536 & 0.3541 & $0.056^{*}$ \\
C6 & $1.0765(5)$ & $0.3358(2)$ & $0.3564(4)$ & $0.0657(10)$ \\
H6A & 1.0058 & 0.3286 & 0.2731 & $0.099^{*}$ \\
H6B & 1.0664 & 0.2953 & 0.4083 & $0.099^{*}$ \\
H6C & 1.1744 & 0.3348 & 0.3570 & $0.099^{*}$ \\
C7 & $0.7666(3)$ & $0.49699(16)$ & $0.2046(3)$ & $0.0314(6)$ \\
C8 & $0.8736(4)$ & $0.4814(2)$ & $0.1455(3)$ & $0.0525(9)$ \\
H8A & 0.8245 & 0.4864 & 0.0570 & $0.079^{*}$ \\
H8B & 0.9119 & 0.4303 & 0.1657 & $0.079^{*}$ \\
H8C & 0.9534 & 0.5177 & 0.1756 & $0.079^{*}$ \\
C9 & $0.5083(3)$ & $0.62416(15)$ & $0.0005(3)$ & $0.0325(6)$ \\
C10 & $0.4915(3)$ & $0.67529(16)$ & $-0.1042(3)$ & $0.0330(6)$ \\
C11 & $0.6000(4)$ & $0.68576(18)$ & $-0.1513(3)$ & $0.0432(7)$ \\
H11 & 0.6886 & 0.6590 & -0.1161 & $0.052^{*}$ \\
C12 & $0.5789(4)$ & $0.73510(18)$ & $-0.2493(3)$ & $0.0425(7)$ \\
H12 & 0.6519 & 0.7408 & -0.2806 & $0.051^{*}$ \\
& & & &
\end{tabular}


Table 2 (continued)

\begin{tabular}{lrrrr}
\hline Atom & $\boldsymbol{x}$ & $\boldsymbol{y}$ & $\boldsymbol{z}$ & $\boldsymbol{U}_{\text {iso }} \boldsymbol{U}_{\text {eq }}$ \\
\hline C13 & $0.4489(3)$ & $0.77581(17)$ & $-0.3004(3)$ & $0.0386(7)$ \\
C14 & $0.3400(4)$ & $0.7662(2)$ & $-0.2530(3)$ & $0.0511(8)$ \\
H14 & 0.2521 & 0.7936 & -0.2871 & $0.061^{*}$ \\
C15 & $0.3615(3)$ & $0.71679(18)$ & $-0.1570(3)$ & $0.0429(7)$ \\
H15 & 0.2878 & 0.7108 & -0.1265 & $0.052^{*}$ \\
C16 & $0.5249(5)$ & $0.8377(2)$ & $-0.4487(4)$ & $0.0572(9)$ \\
H16A & 0.4882 & 0.8743 & -0.5151 & $0.086^{*}$ \\
H16B & 0.5431 & 0.7896 & -0.4805 & $0.086^{*}$ \\
H16C & 0.6152 & 0.8564 & -0.3865 & $0.086^{*}$ \\
\hline
\end{tabular}

filtered and set aside to crystallize for several days, giving yellow block crystals.

\section{Experimental details}

The structure was solved by Direct Methods and refined with the SHELX crystallographic software package [2]. The hydrogen atoms were placed at calculated positions and refined as riding atoms with isotropic displacement parameters.

\section{Discussion}

Hydrozones derived from 2-aceto-pyrazine and their metal complexes have been widely investigated mainly due to their excellent biological activities [3-5]. It is noted that the biological activities of the resulted complexes depend not only on the metal centers but also on the structures of the ligands [3, 4].

In the title crystal structure, the central cadmium(II) ion is five-coordinated to one neutral hydrazone ligand by a $\mathrm{ON}_{2}$ donor set, and two chlorido ligands giving a distorted tetragonal-pyramidal coordination geometry with the geometric index $\tau$ value of 0.032 [6]. In the solid state, the intermolecular $\mathrm{N}-\mathrm{H}$. . O hydrogen bonds link the complexes into a zig-zag chain. Bond lengths and angels are all in the expected ranges [7].

\section{References}

1. Bruker. SMART and SAINT. Bruker AXS Inc., Madison, WI, (USA) 2007.

2. Sheldrick, G. M.: Crystal Structure Refinement with SHELXL. Acta Crystallogr. C71 (2015) 3-8.

3. Xu, J.; Zhou, T.; Xu, Z.-Q.; Gu, X.-N.; Wu, W.-N.; Chen, H.; Wang, Y.; Jia, L.; Zhu, T.-F.; Chen, R.-H.: Synthesis, crystal structures and antitumor activities of copper(II) complexes with a 2-acetylpyrazine isonicotinoyl hydrazone ligand. J Mol. Struct. 1128 (2017) 448-454.

4. Wang, W.-W.; Wang, Y.; Zhang, L.; Song, Y.-F.; Wu, W.-N.; Chen, Z.: Syntheses, crystal structures and DNA-binding properties of $\mathrm{Cu}(\mathrm{II}) / \mathrm{Ni}(\mathrm{II})$ complexes with acylhydrazone ligand bearing pyrazine unit. Chin. J. Inorg. Chem. 35 (2019) 563-568.

5. Hou, X.-F.; Zhao, X.-L.; Zhang, L.; Wu, W.-N.; Wang, Y.: $\mathrm{Co}(\mathrm{II}) / \mathrm{Zn}(\mathrm{II}) / \mathrm{Cu}$ (II) complexes containing hydrazone ligand bearing pyrazine unit: syntheses, crystal structures and fluorescence properties. Chin. J. Inorg. Chem. 34 (2018) 201-205.

6. Ye, X.-P.; Wang, G.-J.; Pan, P.; Zhang, Z.-P.; Wu, W.-N.; Wang, Y.: Syntheses, Crystal structures and biological activities of two $\mathrm{Cu}(\mathrm{II})$ complexes with an acylhydrazone ligand bearing pyrrole unit. Chin. J. Inorg. Chem. 30 (2014) 2789-2795.

7. Kalinowski, D. S.; Sharpe, P. C.; Bernhardt, P. V.; Richardson, D. R.: Structure-activity relationships of novel iron chelators for the treatment of iron overload disease: the methyl pyrazinylketone isonicotinoyl hydrazone series. J. Med. Chem. 51 (2008) 331-344. 